\title{
Robust fault detection for Takagi-Sugeno discrete models: Application for a three-tank system
}

\author{
H. Ghorbel, \\ M. Souissi, \\ M. Chaabane \\ Laboratory of Sciences and Techniques of \\ Automatic control \& computer engineering. \\ National School of Engineering of Sfax, \\ University of Sfax \\ PB 1173, 3038 Sfax, Tunisia.
}

\author{
F. Tadeo \\ Dpt. Systems Engineering, \\ Universidad de Valladolid, \\ 47011 Valladolid, Spain.
}

\begin{abstract}
In this paper, we present a fuzzy observer based on TakagiSugeno (TS) models, to estimate simultaneously the system state and the sensors faults of discrete time nonlinear systems. The method uses the technique of descriptor systems, by considering the sensor faults as auxiliary states variables. More precisely, This paper addresses the problem of index fault detection observer to ensure the sensitivity against the faults. The proposed method is based on the use of the Lyapunov theory to ensure the stability of the system. Necessary and sufficient conditions are obtained in terms of Linear Matrix Inequalities (LMIs), in order to determine the observer gains. An application of the fault estimation method on an hydraulic process with three tanks, using TS models is realized. Simulation and experimental results show the effectiveness of the proposed method.
\end{abstract}

\section{General Terms}

Takagi-Sugeno model, observer design.

\section{Keywords}

Non linear system, TS fuzzy model, descriptor observer, sensor fault estimation, $H_{-}$, LMI, tanks system.

\section{INTRODUCTION}

As it is well known, model based fault detection has attracted several directions in research; among them we can cite the works of $[1,4,9,10,12,25]$. Several methods have been developed to detect and identify sensor faults when they appear $[2,11,13,14,16,17]$. Among these techniques, Takagi-Sugeno (TS) fuzzy model has been used to represent the nonlinear systems [21, 22, 23]. In this context, the nonlinear systems can be represented by a set of linear models interpolated by member ship functions [18]. In order to detect and estimate faults, some results on fault detection observers have been developed in the literature $[5,7,8,15,20]$, such as the fuzzy observer using LMI approach presented in [19], the unknown inputs observer and the nonlinear descriptor estimator [24]. Recently in [25], a method using a descriptor representation, to estimate simultaneously the system state and the sensor faults, has been proposed.

[9] has proposed a sensitive fault observer by using the $H_{-}$norm. In this work, the objective is the synthesis of the sensitive fault observer for a TS discrete time fuzzy model. The TS model is considered with the sensor faults. The synthesis uses the $H_{-}$norm to ensure the sensitivity against the faults. The synthesis conditions are solved in terms of linear matrix inequalities (LMIs).

This paper is organized as follows. In section 2, we present the structure of the TS model and the observer. The synthesis conditions of the robust fault observer are formulated by using the $H_{-}$performance in section 3. Section 4 is devoted to present the tanks laboratory system and to give the corresponding nonlinear model. In section 5, some results obtained experimentally and by simulation are presented to illustrate the efficiency of the proposed method. Finally, section 6 gives some conclusions.

Notation. In this paper, the following notations are used: $X^{T}$ and $X^{-1}$ are the transpose and the inverse of matrix, respectively. $I$ is the identity matrix with appropriate dimension. $P>0$ indicates that the symmetric matrix $P$ is positive definite. The symbol $*$ in a symmetric matrix denotes the transposed block in the symmetric position.

\section{PROBLEM FORMULATION}

\subsection{TS fuzzy model}

We considered a TS model described by fuzzy IF-THEN rules. The $i$ th rule of the model is of the following form:

Rule $i$

$$
\begin{aligned}
& \text { IF } z_{1}(k) \text { is } M_{1 i} \text { and } \ldots z_{q}(k) \text { is } M_{q i} \text { THEN } \\
& \left\{\begin{array}{l}
x(k+1)=A_{i} x(k)+B_{i} u(k) \\
y(k)=\mathrm{C} x(k)
\end{array}\right.
\end{aligned}
$$

where $x(k) \in R^{n}$ is the state vector, $u(k) \in R^{m}$ is the input vector and $y(k) \in R^{p}$ is the measurable output vector. $A_{i}, B_{i}$ and $C$ are matrices with appropriate dimension. $\xi(k)=\left[\xi_{1}(k) \ldots \xi_{q}(k)\right]$ are the premise variables, $M_{1 i} \ldots . M_{q i}$ are the fuzzy sets and $r$ is the number of rules.

Then the TS system is written as follows:

$\left\{\begin{array}{l}x(k+1)=\sum_{i=1}^{r} \mu_{i}(\xi)\left(A_{i} x(k)+B_{i} u(k)\right) \\ y(k)=\mathrm{C} x(k)\end{array}\right.$ 
$\mu_{i}(\xi)=\frac{w_{i}(\xi(t))}{\sum_{i=1}^{r} w_{i}(\xi(t))}, w_{i}(\xi(t))=\prod_{j=1}^{q} M_{j i}(\xi(t))$

Hence, $\mu_{i}(\xi)$ satisfies:

$\sum_{i=1}^{r} \mu_{i}(\xi)=1$ and $\mu_{i}(\xi) \geq 0$ for $i=1, \ldots, r$

In this paper, we considered TS models in discrete time with sensor faults. Then, the system (2) is rewritten in the following form:

$\left\{\begin{array}{l}x(k+1)=\sum_{i=1}^{r} \mu_{i}(\xi)\left(A_{i} x(k)+B_{i} u(k)\right) \\ y(k)=\mathrm{C} x(k)+D_{s} f_{s}(k)\end{array}\right.$

where $f_{s}(k)$ represents the additive sensor fault and $D_{s}$ is a matrix of appropriate dimensions.

In order to estimate the state and the sensor faults, we considered the faults as an auxiliary state of the augmented system.

An augmented system descriptor is then constructed as follows:

$$
\left\{\begin{array}{l}
\bar{E} \bar{x}(k+1)=\sum_{i=1}^{r} \mu_{i}(\xi)\left(\bar{A}_{i} \bar{x}(k)+\bar{B}_{i} u(k)\right)+\bar{D} x_{s}(k) \\
y(k)=\overline{\mathrm{C}} \bar{x}(k)=C_{0} \bar{x}(k)+x_{s}(k)
\end{array}\right.
$$

where:

$$
\begin{aligned}
& \bar{x}(k)=\left[\begin{array}{c}
x(k) \\
x_{s}(k)
\end{array}\right], \quad x_{s}(k)=D_{s} f_{s}(k), \quad E=\left(\begin{array}{cc}
I_{n} & 0 \\
0 & 0
\end{array}\right), \quad \bar{B}_{i}=\left(\begin{array}{c}
B_{i} \\
0
\end{array}\right) \\
& \bar{A}_{i}=\left(\begin{array}{cc}
A_{i} & 0 \\
0 & -I_{n}
\end{array}\right), \quad \bar{D}=\left(\begin{array}{c}
0 \\
I_{p}
\end{array}\right), C_{0}=\left(\begin{array}{ll}
C & 0
\end{array}\right), \bar{C}=\left(\begin{array}{ll}
C & I_{p}
\end{array}\right)
\end{aligned}
$$

In this study, we considered the following observer structure:

$$
\left\{\begin{array}{l}
E z(k+1)=\sum_{i=1}^{r} \mu_{i}(\xi)\left(N_{i} z(k)+\bar{B}_{i} u(k)\right) \\
\hat{\bar{x}}(k)=z(k)+M y(k)
\end{array}\right.
$$

where $z(k) \in R^{n+p}$ is an auxiliary state vector, $\hat{\bar{x}}(k) \in R^{n+p}$ is the estimate of $\bar{x}(k) \in R^{n+p} . \quad E, \quad N_{i} \in R^{(n+p) \times(n+p)}$ and $M \in R^{(n+p) \times p}$ are the design parameters of the observer.

\subsection{Residual generation}

The error estimation and the residual signal are defined as:

$$
\begin{aligned}
& e(k)=\bar{x}(k)-\hat{\bar{x}}(k) \\
& r(k)=V(y(k)-\hat{y}(k))
\end{aligned}
$$

where $V$ is the residual weighting matrix.

\section{Definition:}

Given system (2) and a scalar $\beta>0$, the observer (7) is called $H_{-}$fault detection observer if it is asymptotically stable and the following inequality is satisfied:

$$
r^{T}(k) r(k) \geq \beta^{2} f_{s}^{T}(k) f_{s}(k)
$$

The objective is to design an allowable observer (7) to maximize $\beta$, i.e., a sensitive fault observer. In the following, we determine the error dynamics equation. From the equations (6) and (7), we considered the following system:

$$
\begin{aligned}
& (\bar{E}+E L \bar{C}) \bar{x}(k+1)-E \hat{\bar{x}}(k+1)=\sum_{i=1}^{r} \mu_{i}(\xi) \\
& {\left[\left(\bar{A}_{i}+N_{i} M C_{0}\right) \bar{x}(k)-N_{i} \hat{\bar{x}}(k)+\left(\bar{D}_{s}+N_{i} M\right) x_{s}(k)\right]}
\end{aligned}
$$

In order to determine the observer gains, we consider the following assumption:

$$
\begin{aligned}
& N_{i}=\bar{A}_{i}+N_{i} M C_{0} \\
& \bar{D}=-N_{i} M \\
& E=\bar{E}+E M \bar{C}
\end{aligned}
$$

The error dynamics can be writing as follows:

$$
E \bar{e}(k+1)=\sum_{i=1}^{r} \mu_{i}(\xi) F_{i} \bar{e}(k) \text { for } i=1, \ldots, r
$$

We can easily show that the constraints (12) are checked with the following choice:

$$
\begin{aligned}
& N_{i}=\left(\begin{array}{cc}
A_{i} & 0 \\
-C & -I_{n}
\end{array}\right) \\
& M=\left(\begin{array}{c}
0 \\
I_{p}
\end{array}\right) \\
& E=\left(\begin{array}{cc}
I_{n} & 0 \\
Q C & Q
\end{array}\right)
\end{aligned}
$$

where $Q \in R^{p x p}$ is a full-rank matrix, that is a parameter to determine. By taking account of (14)-(16), the error dynamics becomes:

$$
\bar{e}(k+1)=\sum_{i=1}^{r} \mu_{i}(\xi) S_{i} \bar{e}(k) \quad \text { for } \quad i=1, \ldots, r
$$

where

$$
\begin{aligned}
S_{i} & =E^{-1} N_{i}=\left(\begin{array}{cc}
I_{n} & 0 \\
Q C & Q
\end{array}\right)^{-1}\left(\begin{array}{cc}
A_{i} & 0 \\
-C & -I_{n}
\end{array}\right) \\
& =\left(\begin{array}{cc}
A_{i} & 0 \\
-C A_{i}-Q^{-1} C & -Q^{-1}
\end{array}\right)
\end{aligned}
$$

\section{Remark:}

Therefore, the problem of the observer synthesis comes down to the determination of the matrix $R$ and the residual weighting matrix $V$ such that simultaneously:

- The observation error $e(k)$ aims asymptotically towards zero;

- The residual $r(k)$ provides good fault sensitivity.

We can write in this case:

$r(k)=V\left(C_{0} \bar{e}(k)+D_{s} f_{s}(k)\right)$ 


\section{SYNTHESIS OF}

THE

ROBUST FAULT OBSERVER

In this section, we consider the robust residual problem $r(k)$ via the fault $f(k)$. To attain the objective, the $H_{-}$ performance is used as measurement of the worst case fault sensitivity of the residual generator on the presence of a fault.

\section{Theorem:}

The system (17) and (18) is asymptotically stable and guarantee the performance (10), if there exist matrices $P_{1}>0$ , $P_{2}>0$, matrices $Z_{1}, Z_{2}$ and $V$ and a scalar $\beta>0$, such that the following LMI holds:

$\left[\begin{array}{ccccc}\Lambda_{1 i}+ & A_{i}^{T} C^{T} Z_{2} & 0 & C^{T} V^{T} & C^{T} Z_{2}^{T} \\ 2 G_{1}\left(V, V_{c}^{k}\right) & -P_{2} & 0 & 0 & Z_{2}^{T} \\ * & * & \beta^{2} I+ & D_{s}^{T} V^{T} & 0 \\ * & * & 2 G_{2}\left(V, V_{f}^{k}\right) & & \\ * & * & * & -I & 0 \\ * & * & * & * & -P_{2}\end{array}\right]<0$

where:

$\Lambda_{1 i}=A_{i}^{T} P_{1} A_{i}+A_{i}^{T} C^{T} P_{2} C A_{i}+A_{i}^{T} C^{T} Z_{2} C+C^{T} Z_{2}^{T} C A_{i}-P_{1}$

$G_{1}\left(V, V_{c}^{k}\right)=\left(V_{c}^{k}\right)^{T} V_{c}^{k}-\left(V_{c}^{k}\right)^{T} V C-C^{T} V^{T} V_{c}^{k}$

$G_{2}\left(V, V_{s}^{k}\right)=\left(V_{s}^{k}\right)^{T} V_{s}^{k}-\left(V_{s}^{k}\right)^{T} V D_{s}-D_{s}^{T} V^{T} V_{s}^{k}$

The observer (7) is defined by (14)-(16) with $Q=\left(P_{2}^{-1} Z_{2}\right)^{-1}$

Proof:

The candidate Lyapunov function is defined as:

$V(k)=\bar{e}_{a}^{T}(k) P \bar{e}_{a}(k), P>0$

$$
\begin{aligned}
\Delta V & =V(k+1)-V(k) \\
& =\bar{e}_{a}^{T}(k+1) P \bar{e}_{a}(k+1)-\bar{e}_{a}^{T}(k) P \bar{e}_{a}(k)
\end{aligned}
$$

Thus, $\bar{e}(k+1)=\sum_{i=1}^{r} \mu_{i}(\xi) S_{i} \bar{e}(k)$

Then, $\Delta V=\sum_{i=1}^{r} \mu_{i}(\xi)\left[\bar{e}^{T}\left(S_{i}^{T} P S_{i}-P\right) \bar{e}\right]$

$$
\begin{aligned}
& J_{-}= r^{T}(k) r(k)-\beta^{2} f_{s}^{T}(k) f_{s}(k) \\
&=\left(r^{T}(k) r(k)-\beta^{2} f_{s}^{T}(k) f_{s}(k)-\Delta V(\bar{e})\right)+\Delta V(\bar{e}) \\
&=\left(\left(C_{0} \bar{e}+D_{s} f_{s}\right)^{T} V^{T} V\left(C_{0} \bar{e}+D_{s} f_{s}\right)-\beta^{2} f_{s}^{T} f_{s}-\right. \\
&\left.\sum_{i=1}^{\infty} \mu_{i}(\xi) \bar{e}^{T}\left(S_{i}^{T} P S_{i}-P\right) \bar{e}\right)+\Delta V(\bar{e}) \\
& J_{-}= \sum_{i=1}^{\infty} \mu_{i}(\xi)\left(\begin{array}{c}
\bar{e} \\
f_{s}
\end{array}\right)^{T} \gamma_{i}\left(\begin{array}{c}
\bar{e} \\
f_{s}
\end{array}\right)+\Delta V(\bar{e}) \\
& \text { With } \gamma_{i}=\left(\begin{array}{cc}
-\left(S_{i}^{T} P S_{i}-P\right)+C_{0}^{T} V^{T} V C_{0} & C_{0}^{T} V^{T} V D_{s} \\
* & -\beta^{2} I+D_{s}^{T} V^{T} V D_{s}
\end{array}\right)
\end{aligned}
$$

Consequently, if $\gamma_{i} \geq 0$, we can guarantee $J_{-} \geq 0$, that is,

$\left(\begin{array}{cc}S_{i}^{T} P S_{i}-P-C_{0}^{T} V^{T} V C_{0} & C_{0}^{T} V^{T} V D_{s} \\ * & \beta^{2} I-D_{s}^{T} V^{T} V D_{s}\end{array}\right) \leq 0$

Replacing $S_{i}=\left(\begin{array}{cc}A_{i} & 0 \\ -C A_{i}-R^{-1} C & -R^{-1}\end{array}\right)$ and supposing that $P=\operatorname{diag}\left(P_{1}, P_{2}\right)$, we obtain:

$$
\left(\begin{array}{ccc}
\Delta_{1 i}-C^{T} V^{T} V C & \Omega_{1} & C^{T} V^{T} V D_{s} \\
* & R^{-T} P_{2} R^{-1}-P_{2} & 0 \\
* & * & \beta^{2} I-D_{s}^{T} V^{T} V D_{s}
\end{array}\right) \leq 0
$$

where

$$
\begin{aligned}
\Delta_{1 i} & =A_{i}^{T} P_{1} A_{i}+A_{i}^{T} C^{T} P_{2} C A_{i}+A_{i}^{T} C^{T} Z_{2} C+C^{T} Z_{2}^{T} C A_{i} \\
& -P_{1}+C^{T} R^{-T} P_{2} R^{-1} C \\
\Omega_{1} & =A_{i}^{T} C^{T} P_{2} R^{-1}+C^{T} R^{-T} P_{2} R^{-1}
\end{aligned}
$$

Applying the Schur complement theorem to the LMI (26) and by the following change of variables

$$
V_{s}^{k}=V^{k-1} D_{s} \text { and } V_{c}^{k}=V^{k-1} C \text {, we obtain: }
$$

$$
\left(\begin{array}{cccc}
\Delta_{1 i}+ & & & C^{T} V^{T} \\
2 G_{1}\left(V, V_{c}^{k}\right) & \Omega_{1} & 0 & 0 \\
* & R^{-T} P_{2} R^{-1}-P_{2} & 0 & \\
* & * & \beta^{2} I+ & V D_{s} \\
* & * & 2 G_{1}\left(V, V_{f}^{k}\right) & \\
& & * & -I
\end{array}\right) \leq 0
$$

This LMI (27) can also be rewritten as follows: 


$$
\begin{aligned}
& \left(\begin{array}{cccc}
\Lambda_{1 i}+ & A_{i}^{T} C^{T} P_{2} R^{-1} & 0 & C^{T} V^{T} \\
2 G_{1}\left(V, V_{c}^{k}\right) & -P_{2} & 0 & 0 \\
* & * & \beta^{2} I+ & \\
* & & 2 G_{1}\left(V, V_{f}^{k}\right) & V D_{s} \\
* & * & * & -I
\end{array}\right)+ \\
& \left(\begin{array}{cccc}
C^{T} R^{-T} P_{2} R^{-1} C & C^{T} R^{-T} P_{2} R^{-1} & 0 & 0 \\
* & R^{-T} P_{2} R^{-1} & 0 & 0 \\
* & * & 0 & 0 \\
* & * & * & 0
\end{array}\right) \leq 0
\end{aligned}
$$

Applying Schur complement theorem and assuming that $Z_{2}=P_{2} R^{-1}$, we can get the following $L M I$ easily:

$$
\left[\begin{array}{ccccc}
\Lambda_{1 i}+ & A_{i}^{T} C^{T} Z_{2} & 0 & C^{T} V^{T} & C^{T} Z_{2}^{T} \\
2 G_{1}\left(V, V_{c}^{k}\right) & -P_{2} & 0 & 0 & Z_{2}^{T} \\
* & * & \beta^{2} I+ & D_{s}^{T} V^{T} & 0 \\
* & * & 2 G_{2}\left(V, V_{f}^{k}\right) & & \\
* & * & * & -I & 0 \\
* & * & * & * & -P_{2}
\end{array}\right]<0
$$

\section{THE THREE TANK SYSTEM}

In this section, we present the laboratory system that will be used to test the proposed methodology. Its nonlinear model is also derived.

\subsection{Process description}

Consider the three tanks system shown in Fig 1[6]. The system is composed of three tanks with instrumentation and a deposit . Two pumps permit the water supply of tanks R1 and $\mathrm{R} 2$ by adjustment of the control inputs $u_{1}$ and $u_{2}$, respectively. These tanks $\mathrm{R} 1$ and $\mathrm{R} 2$ are also connected through a manual valve that makes possible to change the dynamics when desired. Two electronic valves EV1 and EV2 are used to fill tank R3, and an electronic valve EV3 is used to evacuate the water.

The water levels are measured with ultrasonic sensors in each tank.

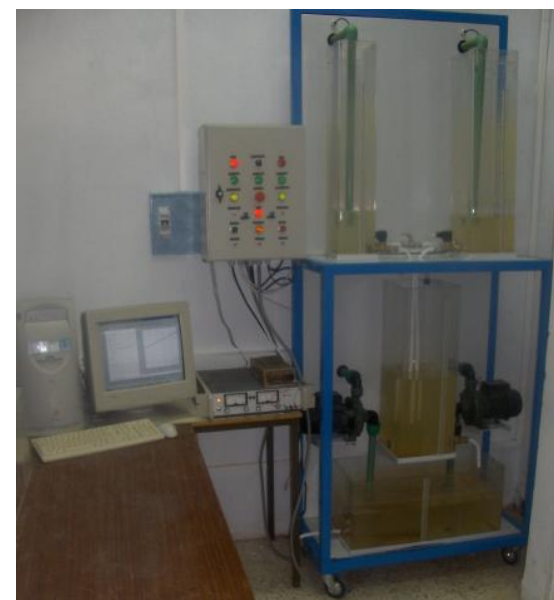

Fig 1: Three tanks system

\subsection{System modeling}

To illustrate the effectiveness of the sensitive fault observer, we consider a nonlinear model of the three tank system as follows:

$$
x(k+1)=\sum_{i=1}^{8} \mu_{i}(\xi)\left(A_{i}(x(k)) x(k)+B_{i} u(k)\right)
$$

The output of the system is:

$$
y(k)=C x(k)+D_{s} f_{s}(k)
$$

where $x(k)=\left(n_{1}(k) n_{2}(k) n_{3}(k)\right)^{T}$

The matrices $A_{i}, B_{i}$ and $C$ are

$$
\begin{aligned}
A_{1} & =\left[\begin{array}{ccc}
1-T_{e} p_{1} \beta_{1} & 0 & 0 \\
0 & 1-T_{e} p_{2} \beta_{2} & 0 \\
T_{e} p_{1} \beta_{1} & T_{e} p_{2} \beta_{2} & 1-T_{e} p_{3} \beta_{3}
\end{array}\right] ; \\
A_{2} & =\left[\begin{array}{ccc}
1-T_{e} p_{1} \beta_{1} & 0 & 0 \\
0 & 1-T_{e} p_{2} \beta_{2} & 0 \\
T_{e} p_{1} \beta_{1} & T_{e} p_{2} \beta_{2} & 1-T_{e} p_{3} \alpha_{3}
\end{array}\right] ; \\
A_{3} & =\left[\begin{array}{ccc}
1-T_{e} p_{1} \beta_{1} & 0 & 0 \\
0 & 1-T_{e} p_{2} \alpha_{2} & 0 \\
T_{e} p_{1} \beta_{1} & T_{e} p_{2} \alpha_{2} & 1-T_{e} p_{3} \beta_{3}
\end{array}\right] ; \\
A_{4} & =\left[\begin{array}{ccc}
1-T_{e} p_{1} \beta_{1} & 0 & 0 \\
0 & 1-T_{e} p_{2} \alpha_{2} & 0 \\
T_{e} p_{1} \beta_{1} & T_{e} p_{2} \alpha_{2} & 1-T_{e} p_{3} \alpha_{3}
\end{array}\right] ;
\end{aligned}
$$$$
A_{5}=\left[\begin{array}{ccc}
1-T_{e} p_{1} \alpha_{1} & 0 & 0 \\
0 & 1-T_{e} p_{2} \beta_{2} & 0 \\
T_{e} p_{1} \alpha_{1} & T_{e} p_{2} \beta_{2} & 1-T_{e} p_{3} \beta_{3}
\end{array}\right] \text {; }
$$$$
A_{6}=\left[\begin{array}{ccc}
1-T_{e} p_{1} \alpha_{1} & 0 & 0 \\
0 & 1-T_{e} p_{2} \beta_{2} & 0 \\
T_{e} p_{1} \alpha_{1} & T_{e} p_{2} \beta_{2} & 1-T_{e} p_{3} \alpha_{3}
\end{array}\right]
$$$$
A_{8}=\left[\begin{array}{ccc}
1-T_{e} p_{1} \alpha_{1} & 0 & 0 \\
0 & 1-T_{e} p_{2} \alpha_{2} & 0 \\
T_{e} p_{1} \alpha_{1} & T_{e} p_{2} \alpha_{2} & 1-T_{e} p_{3} \alpha_{3}
\end{array}\right]
$$$$
B=\left[\begin{array}{cc}
p_{11} & 0 \\
0 & p_{22} \\
0 & 0
\end{array}\right] ; C=\left[\begin{array}{ccc}
1 & 0 & 0 \\
0 & 1 & 0 \\
0 & 0 & 1
\end{array}\right]
$$

with

$$
\begin{aligned}
& \alpha_{1}=1 / \operatorname{sqrt}\left(n_{1} \max \right) ; \\
& \alpha_{2}=1 / \operatorname{sqrt}\left(n_{2} \max \right) ; \\
& \alpha_{3}=1 / \operatorname{sqrt}\left(n_{3} \max \right) ; \\
& \beta_{1}=1 / \operatorname{sqrt}\left(n_{1} \min \right) ; \\
& \beta_{2}=1 / \operatorname{sqrt}\left(n_{2} \min \right) ; \\
& \beta_{3}=1 / \operatorname{sqrt}\left(n_{3} \min \right) .
\end{aligned}
$$

and $B_{1}=B_{2}=B_{3}=B_{4}=B_{5}=B_{6}=B_{7}=B_{8}=B$ 
The residual weighting matrix $V$ is

$V=\left[\begin{array}{ccc}20.5160 & 0.0542 & -0.1315 \\ 0.0541 & 20.4744 & -0.1081 \\ -0.1316 & -0.1082 & 20.6563\end{array}\right]$

The considered faults sensors are $f_{s}=\left(\begin{array}{l}f_{s 1} \\ f_{s 2} \\ f_{s 3}\end{array}\right)$

$f_{S 1}=\left\{\begin{array}{cc}0 & \mathrm{k}<60 \\ 0.15^{*} k & 60 \leq \mathrm{k}<80 \\ 0 & \mathrm{k} \geq 80\end{array}\right.$

$f_{s 2}=\left\{\begin{array}{cc}0 & \mathrm{k}<60 \\ 2 * \sin (5 *(k-6)) & 60 \leq \mathrm{k}<80 \\ 0 & \mathrm{k} \geq 80\end{array}\right.$

$f_{s 3}=\left\{\begin{array}{cc}0 & \mathrm{k}<60 \\ 0.1 *(k-2) & 60 \leq \mathrm{k}<70 \\ 0 & \mathrm{k} \geq 70\end{array}\right.$

\section{SIMULATED AND EXPERIMENTAL RESULTS}

Some simulation results are shown in Figures 2-3 via the proposed sensitive fault observer. First, the estimations of the states are plotted in Fig 2.
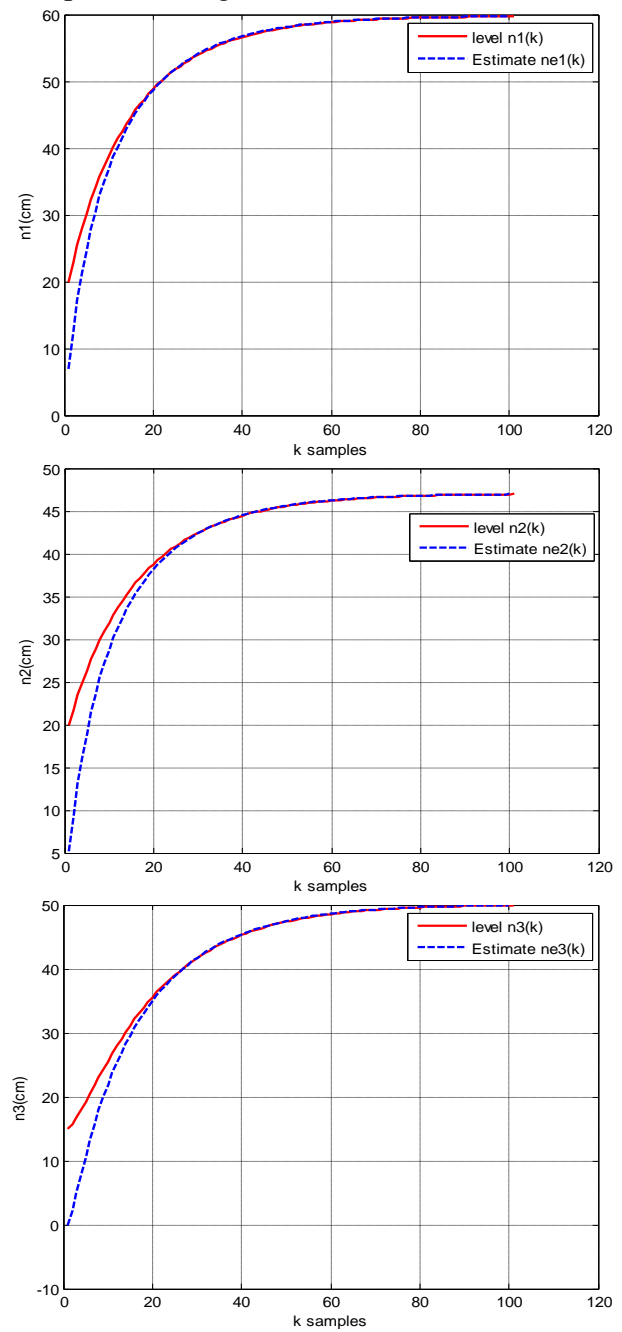

Fig 2: Evolution of the tanks levels and their estimates
Fig 3 shows the sensor faults and their estimates.
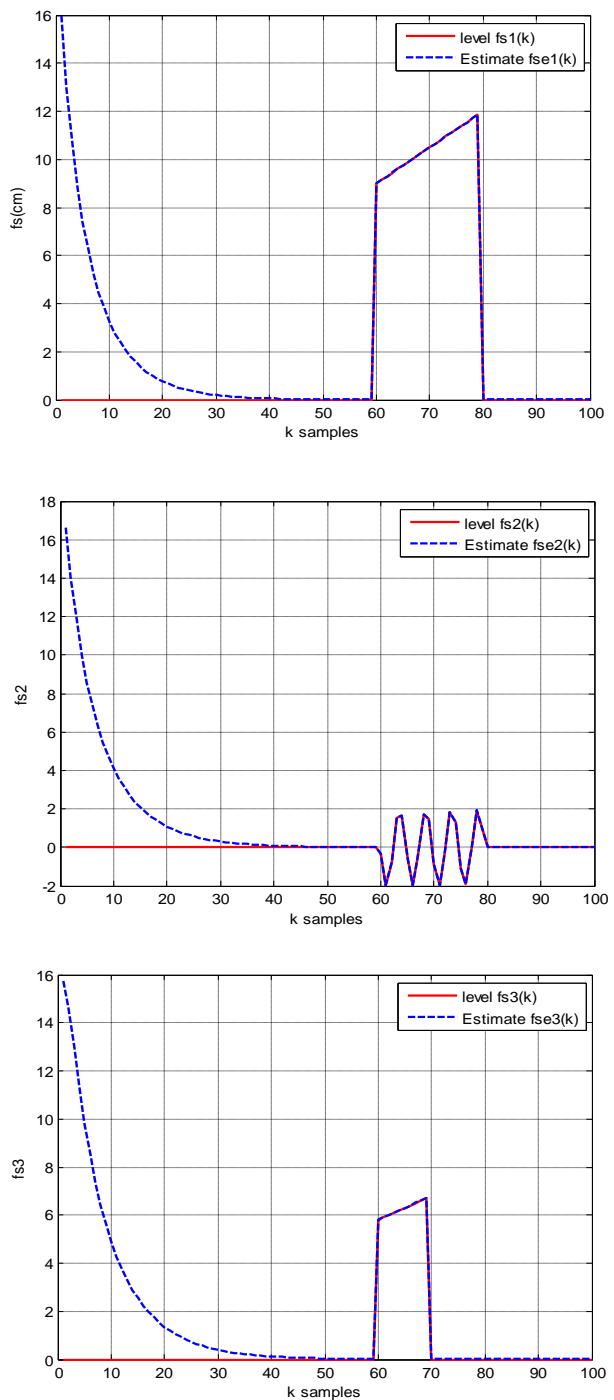

Fig 3: Evolution of the faults and their estimation

Some experimental results are shown in Fig 4-5. Fig 4 compares the evolution of the levels in the three tanks with their estimations: it can be seen that the observer provides adequate estimation of the states, even in the presence of faults (presented in Fig 5).

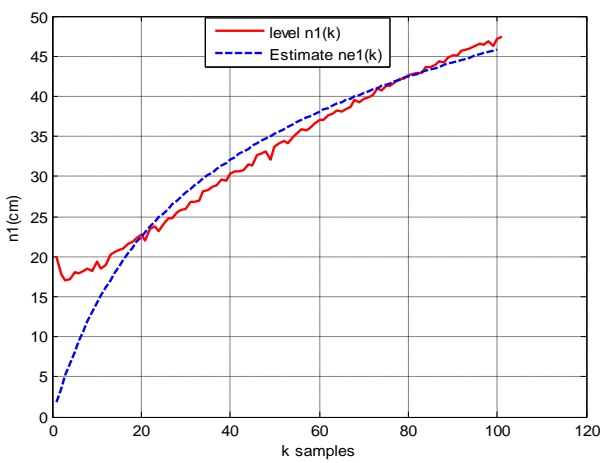



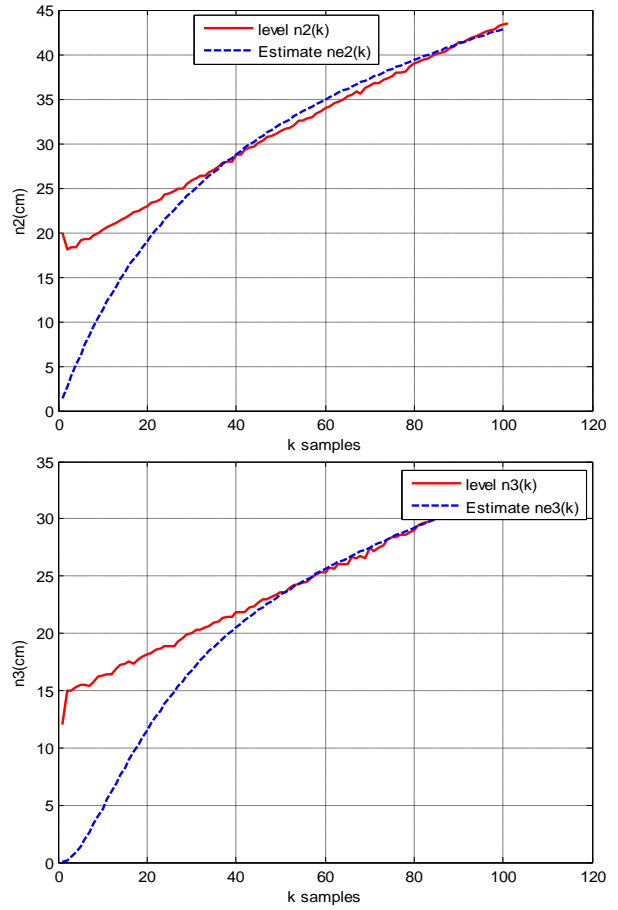

Fig 4: Evolution of the tanks levels, and their estimates
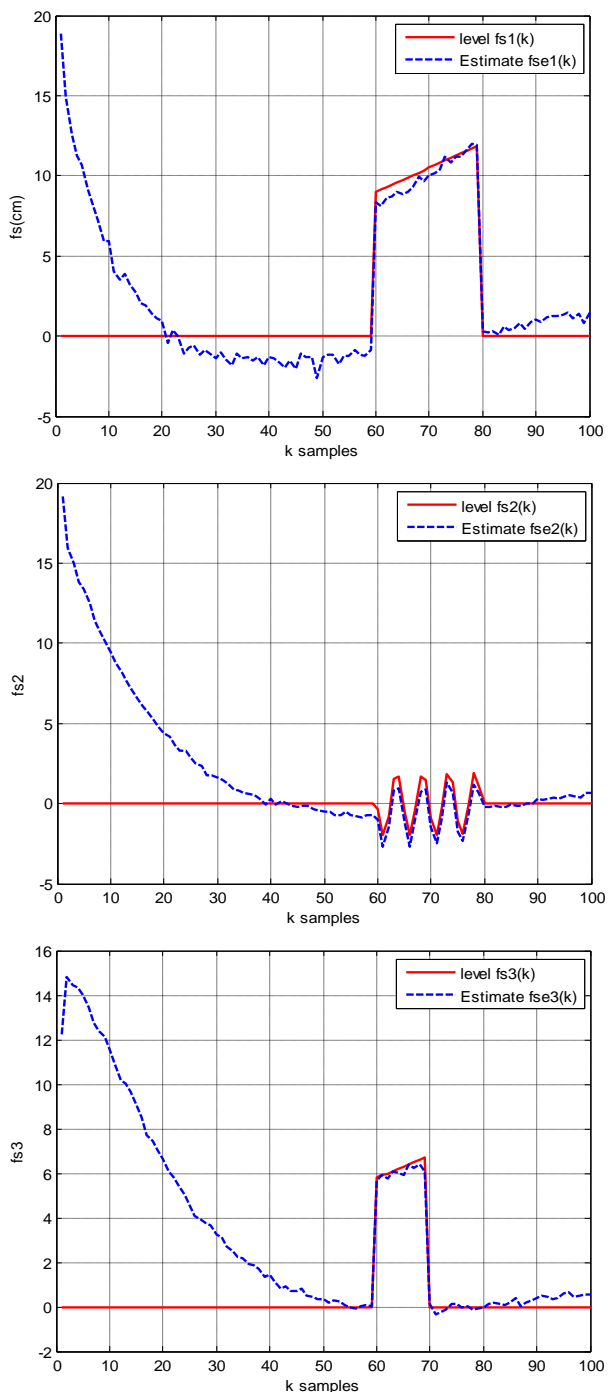

Fig 5: Evolution of the faults and their estimation
In summary, by examining the trajectories presented in Fig 25 , we can confirm that the observer sensitive to the fault gives a good performance. The proposed method supplies adequate estimations of the state of the system and the faults sensors. The obtained results show the efficiency of the proposed approach.

\section{CONCLUSION}

In this paper, the design of an $H_{-}$fault detection observer for Takagi-Sugeno discrete-time systems is investigated. This approach allows ensuring the sensitivity via the sensor faults, by using a descriptor systems technique. Sufficient conditions for the existence of a robust observer are expressed in terms of Linear Matrix Inequalities (LMI), by using a quadratic Lyapunov function. Indeed, the design condition ensures the convergence of the observer, guaranteeing an $H_{-}$ performance.

Finally, we have applied the proposed approach on a laboratory plant (a three tanks system). The simulation and experimental results show that the proposed observer gives good estimates for the system states and the sensor faults. Thus, we can confirm the effectiveness of the proposed method.

\section{ACKNOWLEDGMENTS}

We thank the ministry of higher education and scientific research of Tunisia and AECID (A/030410/10 and AP/039213/11) for funding this work.

\section{REFERENCES}

[1] Khedher, A., Benothman, K., Maquin, D. and Benrejeb, M., "An approach of faults estimation in Takagi-Sugeno fuzzy systems". 8th ACS/IEEE International Conference on Computer Systems and Applications, AICCS 2010, Hammamet, Tunisia, May 2010.

[2] Marx, D., Koenig, B. and Ragot, J., "Design of observers for Takagi- Sugeno descriptor systems with unknown inputs and application to fault diagnosis". IET Control Theory and Applications, vol. 1, no. 5, pp. 1487-1495, 2007.

[3] Castillo-Toledo, B., and Anzurez-Marin, J., "Model-based fault diagnosis using sliding mode observers to TakagiSugeno fuzzy model". IEEE Congress on Intelligent Control, Limassol, Cyprus, 2005.

[4] Ichalal, D., Marx, D. B., Ragot, J. and Maquin, D., "Observer based actuator fault tolerant control for nonlinear Takagi-Sugeno systems: an LMI approach". 18th Mediterranean Conference on Control and Automation, MED'10, Athens, Greece, June 23-25, 2010.

[5] Wang, H., Wang, J., Liu, J. and Lam, J., "Iterative LMI approach for robust fault detection observer design". In Proceedings of the 42nd IEEE Conference on Decision and Control, Maui, Hawaii, USA, December 2003.

[6] Ghorbel, H., Ghamgui, M., Souissi, M. and Chaabane, M., "Modelling and robust control for Three Tanks System using fuzzy approach". Conference JTEA. 27 March 2010 .

[7] Jaimoukha, I., and Li, Z. "A matrix factorization solution to the $\mathrm{H} / \mathrm{H}_{\infty}$ fault detection problem," Automatica, vol. 42, no. 11, pp. 1907-1912, November 2006. 
[8] Liu, J., Wang, J. L and Yang, G. "An LMI approach to minimum sensitivity analysis with application to fault detection," Automatica, vol. 41, no. 11, pp. 1995-2004, 2005.

[9] Guoa, J., Huanga, X. and Cui, Y. "Design and analysis of robust fault detection filter using LMI tools". Computers and Mathematics with Applications, 57(11-12):17431747, June 2009.

[10] Chadli, M., Akhenak, A., Ragot, J. and Maquin, D., "On the design of observer for unknown inputs fuzzy models". International Journal of Automation and Control, 2(1):113-125, 2008.

[11] Blanke, M., Kinnaert, M., Lunze, J. and Staroswiecki, M., " Diagnosis and FTC". Springer-Verlag, London, 2006.

[12] Bouattour, M., Chadli, M., El.Hajjaji, A. and Chaabane, M., "State and faults estimation for T-S models and application to fault diagnosis". 7th IFAC Symposium on Fault Detection, Supervision and Safety of Technical Processes, SAFEPROCESS'09, Barcelona, July 302009.

[13] Bouattour, M., Chadli, M., El.Hajjaji, A. and Chaabane, M., " $\mathrm{H}_{\infty}$ sensor faults estimation for T-S models using descriptor techniques: Application to fault diagnosis". In IEEE International Conference on Fuzzy Systems, Jeju Island, Korea, 20-24 August 2009, pp. 251-255.

[14] Chadli, M., Akhenak, A., Ragot, J. and Maquin, D., "State and unknown input estimation for discrete time multiple model". Journal of the Franklin Institute, vol. 346, no. 6, pp. 593-610, 2009.

[15] Zhong, M., Ding, S., Lam, J. and Wang, H., "An LMI approach to design robust fault detection filter for uncertain LTI systems," Automatica, vol. 39, pp. 543$550,2003$.

[16] Patton, R., Frank, P. M. and Clark, R., "Issues of fault diagnosis for dynamic systems". Springer-Verlag, London, 1999.
[17] Patton, R. and Chen, J., "Obeserver based fault detection and isolation : robustness and applications". Control Eng. Practice, vol. 5, no. 5, pp. 671-682, 1997.

[18] Orjuela, R., Marx, D. B., Ragot, J. and Maquin, D., "Diagnostic des systèmes non linéaires par une approche multimodèle". 6th Conférence Internationale Francophone d'Automatique, CIFA 2010, Nancy, France, June 2-4, 2010

[19] Patton, R., and Chen, J., "Fuzzy Observers for Non-linear Dynamic Systems Fault Diagnosis". IEEE Conference on Decision asd Control Tampa, Florida USA, 1998.

[20] Nguang, S., Shi, P. and Ding, S., "Fault detection for uncertain fuzzy systems: an LMI approach". IEEE Tr. Fuzzy Systems, vol. 15, no. 6, pp. 1251-1262, 2007.

[21] Takagi, T., and Sugeno, M., "Fuzzy identification of systems and its applications to modeling and control". IEEE Trans. Syst., Man, Cybern., vol. SMC-15, no. 4, pp. 116-132, Aug. 1985

[22] Taniguchi, T., Tanaka, K. and Wang, H. O., "Fuzzy descriptor systems and nonlinear model following control”. IEEE Trans. Fuzzy Syst., vol. 8, no. 4, pp. 442452, Aug. 2000.

[23] Bouarar, T., Guelton, K., Mansouri, B. and Manamanni, N., "LMI Stability Conditions for Takagi-Sugeno Uncertain Descriptors". International Conference on Fuzzy Systems (FUZZ-IEEE). London, UK, July 2007.

[24] Gao, Z., and D. W. C., Ho., "State/Noise Estimator for Descriptor Systems with Application to Sensor Fault Diagnosis". IEEE Transactions on Signal Processing, vol. 54, no. 4, 2006.

[25] Gao, Z., Shi, X. and Ding, S. X., "Fuzzy statedisturbance observer design for T-S fuzzy systems with application to sensor fault estimation". IEEE Transactions on SMC-part B, vol. 38, no. 3: 875 - 880, 2008. 